\title{
Desequilibrios informativos en la prensa deportiva española en Internet. Análisis de Marca, As, Mundo Deportivo y Sport
}

\section{Information imbalances in the Spanish sports press on Internet. Analysis of Marca, As, Mundo Deportivo y Sport}

\author{
Leopoldo Abad Alcalá. Universidad CEU San Pablo (abad.fhm@ceu.es)
}

Ignacio Calvo García. Doctorando de la Universidad CEU San Pablo (ignaciocalvogarcía@gmail.com)

Recibido: 9-IX-2015 - Aceptado: 22-IX-2015

Resumen:

Este artículo presenta los resultados de un estudio empírico cuantitativo sobre los deportes tratados en sus principales informaciones por los más significativos diarios deportivos españoles en su edición de Internet. Se plantea y se discute la tesis que entiende que la información deportiva en Internet prima los contenidos sobre fútbol minimizando la información sobre otros deportes, y especialmente publica contenidos sobre el Real Madrid y el F.C. Barcelona. Se han analizado las 30 primeras noticias de las ediciones digitales de los diarios deportivos Marca, As, Mundo Deportivo y Sport durante 61 días de forma no consecutiva, examinado 1.830 contenidos de cada uno de los diarios, lo que asciende a un total de 7.320 informaciones estudiadas. En este sentido, las conclusiones obtenidas confirman dicha tesis mostrando cómo la información sobre fútbol acapara casi un 92\% del total de informaciones y las noticias sobre el Real Madrid y el Barcelona un 65,25\% del total de informaciones.

Palabras clave:

Prensa deportiva; información; análisis cuantitativo; fútbol; deporte.

Abstract:

This article presents the results of a quantitative empirical study on sports treated in the main information for the most significant Spanish sports daily in its online edition. This thesis presents and discusses that online sports newspapers give preference to information about football and minimize the information on other sports, and they especially publish contents about the Real Madrid and FC Barcelona. We analyzed top 30 news for 61 days non-consecutive of online editions of sports dailies (Marca, As, Mundo Deportivo and Sport). We examined 1,830 contents each day, amounting to total 7,320 news studied. In this sense, the conclusions confirm our thesis that show how 92\% of total sports information is about football, and news about Real Madrid and Barcelona add a 65.25\% of all sports information.

Keywords:

Sports newspapers; information; quantitative analysis; football; sport. 


\section{Introducción}

La prensa deportiva es la especialidad periodística con un mayor seguimiento en España. Según los últimos datos del Estudio General de Medios (EGM) (Octubre 2014-Mayo 2015), el diario Marca tuvo una media de lectores diarios de 2.344.000, mientras que As tuvo una media de 1.239.000 lectores diarios, Mundo Deportivo 553.000 lectores diarios y Sport 469.000 lectores diarios; ocupando las posiciones 1..$^{\mathrm{a}}$, 3. ${ }^{\mathrm{a}}, 7 .^{\mathrm{a}}$ y 10. ${ }^{\mathrm{a}}$, respectivamente, del total de medios impresos. Si nos atenemos a su seguimiento por Internet, Marca es, al margen de Youtube, la página Web más seguida en nuestro país. Para el mismo periodo anterior, el EGM establece que Marca.com tiene un total de 5.439 .000 visitantes únicos diarios (sólo superado por los 22.532.000 visitantes únicos diarios de Youtube). En referencia al seguimiento de los otros medios deportivos en Internet, As.com tiene 2.556.000 visitantes únicos diarios, Mundo Deportivo 1.309.000 y Sport 1.221.000. La web de medición de tráfico en la red Alexa.com, coincide en darle el liderazgo a marca.com, en la comparativa de los cuatro diarios deportivos que estamos analizando, como la página más visitada. En su clasificación global de webs más visitadas, la sitúa alrededor de la posición $400^{\text {a }}$ en los meses de enero a junio de 2015. As linda entre la posición $950^{\mathrm{a}}$ y $1.000^{a}$; mientras que Mundo Deportivo y Sport se encuentran en una línea pareja, entre la posición 2.100a, como se aprecia en el siguiente gráfico:

Grafico 1: Tráfico web mundial de la prensa deportiva española

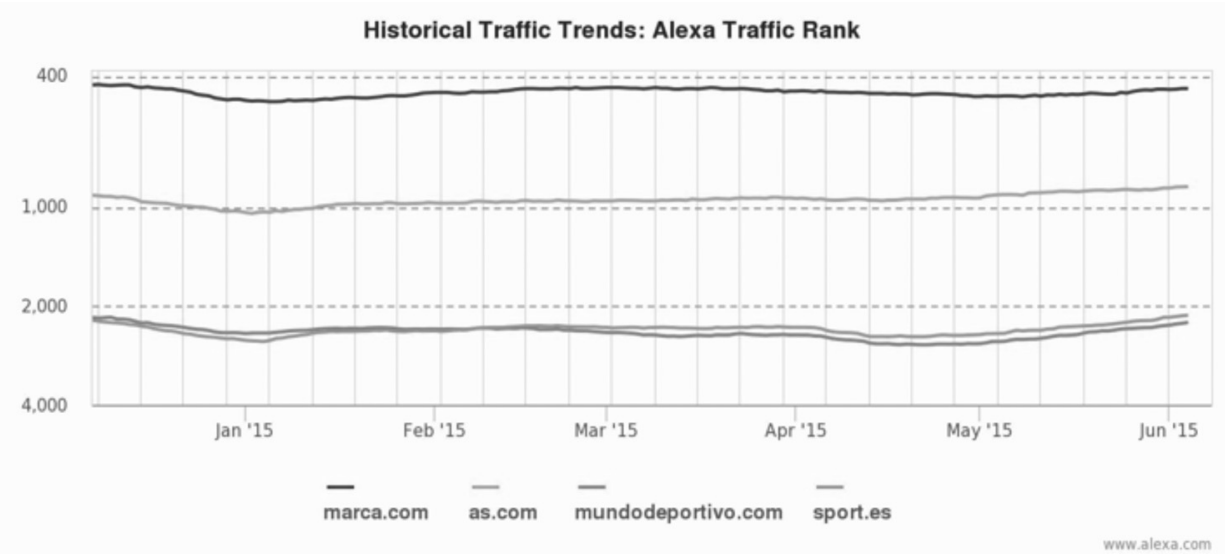

Fuente: Alexa.com

No obstante, a pesar de tratarse de la modalidad periodística más popular en nuestro país, los estudios sobre la prensa deportiva no han sido tan profusos como cabía esperar, a pesar de la atención que concita y el importante reclamo que supone para las audiencias que se traduce en "la especial dedicación que se presta a esta materia en los telediarios, periódicos y medios digitales de contenido general y especializado" (Gil y Romero, 2012: 57), llegándose a considerar que "la 
presencia de las noticias de futbol (y los demás deportes) en los periódicos de información general revelaría que su tratamiento, presentación y valoración son preferentes" (Artells, 1996: 151).

Los principales estudios sobre la prensa deportiva han estado más centrados en su caracterización como prensa especializada (Warren: 1975; Esteve y Fernández del Moral: 1999; Herrero: 2010; entre otros), o han abordado el periodismo deportivo desde una perspectiva lingüística, coincidiendo todos ellos en la existencia de una cierta relajación en el cumplimiento de las normas lingüísticas en aras de una mayor espectacularidad y expresividad que conecte con los sentimientos del lector/oyente/televidente (Núñez Ladeveze: 1991; Lázaro Carreter: 1994; Hernández Alonso; 2003; Castañón: 1993 y 2004; Sobrados: 2009). También se ha analizado el periodismo deportivo desde una aproximación histórica aunque no existe una obra general sobre la historia de la prensa deportiva española, sino aproximaciones parciales en obras sobre la prensa deportiva en general (Relaño: 2004), sobre la prensa deportiva madrileña (Altabella: 1987), o sobre medios específicos como Marca (Toro: 2008). Quizá las obras de referencia en la materia sean los trabajos de Antonio Alcoba sobre la prensa deportiva (1999) y el periodismo deportivo (2005), que abordan su evolución histórica en el marco de su aproximación omnicomprensiva a este tipo de medios. También es destacable la obra de Rojas sobre el periodismo deportivo de calidad (2011) quien estudia en profundidad la caracterización del periodismo deportivo en España y resto de países de habla hispana aportando una reflexión académica sobre las carencias de esta especialidad informativa y concluyendo con una propuesta de libro de estilo panhispánico para informadores deportivos. Otras aproximaciones a la prensa deportiva se han realizado desde su vinculación con la violencia (Barrero: 2008 y 2009), desde su valoración como espectáculo incidiendo en su aspectos económicos (García Candau: 1999) o desde una perspectiva regional (Jones: 1996).

Antes esta situación, creemos necesario realizar una investigación que abordara desde el análisis de contenido las características principales de la prensa deportiva en Internet con la finalidad de valorar su adecuación a criterios de calidad informativa. En concreto, la investigación se centra en los cuatro grandes diarios deportivos de mayor difusión a nivel nacional: Marca, As, Mundo Deportivo y Sport.

Una primera aproximación intuitiva a la prensa deportiva nos muestra una 'futbolización' de la información deportiva que se encuentra copada por noticias sobre fútbol y especialmente sobre el Real Madrid y el F.C. Barcelona, en consonancia con lo indicado por Herrero al afirmar que "cuando hablo de "futbolización del espacio público, me refiero a la presencia y predominio de la información relativa al fútbol en los medios de comunicación” enumerando a continuación ejemplos que corroboran tal aseveración (2010:141-142), opinión compartida por diversos autores (Alcoba, 1980: 120; Berrocal y Rodríguez Maribona, 1998:198-199; Rojas, 2012: 310).

Para la comprobación de esta hipótesis vamos a realizar un primer análisis de carácter cuantitativo de la prensa deportiva en Internet. La elección de las versiones digitales de los periódicos deportivos se fundamenta en dos razones, una de carácter cuantitativo y otra de carácter cualitativo. En virtud de la primera, puede comprobarse como las versiones digitales 
de los diarios impresos deportivos adquieren una gran preeminencia entre las preferencias de los usuarios de Internet, como afirma la Asociación Española de Diarios de España (AEDE) al reconocer que "existe un cambio de hábito de lectura en el lector tradicional de prensa en papel, que migra cada vez más hacia los soportes online y los distribuidores y agregadores de noticias" (AEDE, 2014: 14). Según el Libro Blanco de 2014 de esta Asociación, con respecto a la información en Internet:

“de las tres modalidades de prensa digital-general, deportiva y económica-, la de información general es la que más usuarios únicos logra reunir: 18.341.000 frente a la información deportiva, que alcanza 14.556.000, y a la información económica con 7.584.000. Sin embargo, es en el número de páginas vistas donde la información deportiva gana la batalla con un total de 296,7 millones frente a los 74,9 millones de la prensa digital de información general y las 76 de información económica. En cuanto al promedio de minutos de lectura al día, la prensa de información general acumula un total de 55 minutos, mientras que la información deportiva y económica llegan a 39 y 13 minutos respectivamente. [...] El total de lectores de diarios digitales se sitúa en 7.361.000, con un crecimiento muy elevado, si consideramos que en 2001 tan solo se registraban 406.000. El reparto por sexos de los lectores de prensa, varía sensiblemente entre la digital, 64,6\% hombres y 35,4\% mujeres, y la impresa, 59,4\% hombres y 40,6\% mujeres, en el año 2012" (AEDE, 2014: 95-96).

En virtud de la segunda razón, consideramos que la prensa en Internet, y especialmente la prensa deportiva por su cualidades lúdicas y por adaptarse mejor este medio a las necesidades informativas del deporte por su hipertextualidad, adquirirá en un futuro mayor preponderancia sobre la prensa escrita y los medios audiovisuales. A las razones ya aducidas sobre el desarrollo del periódico electrónico interactivo multimedia -la incapacidad de los periódicos de aumentar e incluso mantener sustancialmente el número de lectores; aumento de la información; diversificación de la actividad empresarial comunicativa; escasez de papel y aumento de la sensibilidad ecologista; así como los avances técnicos- (Diaz Noci y Meso, 1999: 21-22), se unen una serie de características que creemos potencian el desarrollo de la prensa deportiva en Internet (Berrocal y García, 2001: 235):

1) Multimedia: prensa, radio y televisión ofertan en Internet texto, audio y vídeo.

2) Interactivo: los internautas pueden enviar sus opiniones de forma inmediata, por correo electrónico u, hoy ya, a través de las redes sociales. También tienen la posibilidad de participar en debates, foros, encuestas e incluso chats.

3) Información personalizada: el individuo puede programar el medio para encontrar con rapidez la información que le interesa, mediante unos parámetros que el usuario establece de antemano.

4) Nueva redacción periodística: se multiplican las informaciones, aumentan las fuentes y cambian los géneros, la estructura y los estilos de redacción. Los periodistas del siglo XXI también han de ser multimedia. 
5) Variaciones en el formato: el soporte del medio cambia y facilita la búsqueda de contenidos.

6) Nueva dimensión en el diseño: el diseño online ha de invitar al usuario a interaccionar, con lo que ha de traspasar la línea de consecución de la pura belleza estética. Ha de lograr una fácil y útil navegación y evitar que el navegante se pierda.

7) La inmediatez: la oportunidad de informar de manera instantánea sitúa al medio digital con ventaja frente a la prensa en papel.

8) Enlaces: los medios en la red ofertan enlaces hacia motores de búsqueda en Internet e hipervínculos a direcciones que complementan la información.

9) Servicios: ofrece variedad de oportunidades para acceder a la información: hemeroteca, obtener por correo electrónico una selección de titulares e incluso comprar en las tiendas virtuales del medio.

10) Publicidad: la publicidad también se ha adaptado a los medios de comunicación digitales. Se utiliza con frecuencia el banner (bandera), aunque en la actualidad la publicidad en Internet ha conseguido un nivel notable de sofisticación.

De este modo, la tesis inicial que pretendemos demostrar en el presente trabajo es la existencia de sobreinformación sobre fútbol en los contenidos de los medios deportivos digitales y el abandono del tratamiento de otros deportes, lo que supondría como consecuencia de esta "futbolización" de la información que:

"la necesidad de satisfacer una demanda de información diaria sobre este deporte [el fútbol] deviene en contenidos más triviales y ligeros, que carecen de un tratamiento informativo adecuado, ya sea por un sobredimensionamiento de los acontecimientos ya sea por la inclusión de asuntos que tienen más que ver con otro tipo de programas [...] que con la temática puramente deportiva. [...] La tendencia hacia la espectacularización de la información deportiva en televisión, que está estrechamente ligada a la adopción de técnicas sensacionalistas y determina los rasgos estilísticos y el lenguaje empleado a la hora de transmitir los hechos al público, se caracteriza por la magnificación visual y la trivialización de los contenidos, lo que de forma inexorable provoca una pérdida de calidad en el producto informativo" (Rojas, 2011:310).

Esta tesis podría ser considerada como un indicador objetivo de la situación actual de la prensa deportiva y de sus características. Esta primera aproximación al estudio de la prensa deportiva en Internet mostrará datos puramente cuantitativos sobre la presencia de los diversos deportes y específicamente sobre algunos clubes de fútbol. En fases posteriores de esta investigación se pretende un estudio sobre los géneros más empleados en la prensa deportiva y se concluirá con un estudio de carácter cualitativo sobre las posibles disfunciones informativas en la prensa deportiva digital en España. 
Los objetivos de esta investigación pueden dividirse en generales y específicos. Como objetivo general pretendemos analizar el grado de atención que prestan los principales diarios deportivos en sus ediciones en Internet a los distintos deportes y comprobar si existe, como se intuye, una presencia porcentual desmesurada del futbol. Como objetivos específicos, pretendemos en primer lugar probar si dentro de esa información dominante sobre fútbol, esta a su vez está copada por las noticias relacionadas con el Madrid y el Barcelona. En segundo lugar, pretendemos analizar el tratamiento que cada medio deportivo analizado a cada uno de los deportes, para observar si existen diferencias significativas. El tercero de los objetivos de la investigación será comprobar si existe un tratamiento informativo predominante de los diarios deportivos al equipo de su ciudad y si existen divergencias reveladoras entre ellos en esta atención.

El trabajo de campo, consistirá en analizar durante un periodo de 61 días los cuatro portales web deportivos más importantes de España (Marca, As, Mundo Deportivo y Sport) y extraer conclusiones basadas en datos cuantitativos. Esto se convertirá en los cimientos para establecer conclusiones y comprobar la hipótesis que tratamos de demostrar y si por tanto la tesis que de ella se deriva se confirma. Como es evidente, resulta físicamente inabarcable el estudio diario de estos portales informativos al completo, así que como representación de la misma y porque implica la jerarquización del diario (es decir, lo que considera más trascendente), utilizaremos como base la portada de cada uno de estos portales web, en concreto, los 30 primeros contenidos que aparezcan en ella, pues analizarla al completo, por ese mismo motivo de exceso de información, resultaría prácticamente imposible. Por tanto, los resultados de nuestro estudio estarán basados en 7.320 contenidos, una muestra más que representativa para comprobar nuestra hipótesis inicial.

\section{Metodología}

El diseño de nuestro trabajo de campo se clasifica en la categoría de investigación longitudinal de panel, es decir, seguimos las portadas de la prensa deportiva durante un tiempo concreto y de manera no correlativa. De esta manera, hallamos si la hipótesis de partida se mantiene o, por el contrario, simplemente se trata de un hecho circunstancial (Hernández, Hernández y Baptista, 2008: 274). Con respecto al trabajo de campo, nuestra metodología (Campbell y Stanley: 1966; Duverger: 1974; Ferman y Levin: 1979; Sierra: 1991; Padua: 2000; Rojas: 2001; Grinell: 2004) se basa en un enfoque cuantitativo. Gracias a la recolección de datos, obtenemos unos resultados cuantificables que nos sirven para probar la hipótesis inicial tomando como base la medición numérica y un análisis estadístico, con el que pretenderemos comprobar patrones de comportamiento y se prueban otras hipótesis secundarias o teorías. En nuestro caso, el análisis puramente cuantitativo lo hemos aplicado para la hipótesis en la que nos planteamos si existe un exceso de información relacionada con el fútbol en detrimento de otros deportes y especialmente sobre algunos clubes determinados. De este modo hemos escogido un tema concreto y delimitado sobre el que planteamos una hipótesis antes de recolectar los datos. 
Pérez Serrano resume los fundamentos de la metodología de la investigación cuantitativa de los autores citados anteriormente. De manera compendiada, sostiene que el modelo más comúnmente utilizado consta de siete fases, que hemos seguido: 1) Recogida de datos de acuerdo con la especificación de variables realizada y el diseño de la investigación. 2) Tabulación de datos. 3) Representación de distribuciones. 4) Hipótesis estadísticas de acuerdo con la investigación propuesta. 5) Prueba de hipótesis estadísticas. 6) Inferencia respecto a la hipótesis de trabajo. 7) Posible afirmación, ampliación o cambio de la teoría inicial (Pérez Serrano, 2008: 108).

Pero en este análisis de contenido cuantitativo, como propone Cea D'Ancona, no nos limitamos a cuantificar el contenido manifiesto de la comunicación, sino que también abordamos la interpretación del contenido latente; es decir, realizamos un ejercicio de pluralidad analítica en el que cruzamos datos para extraer conclusiones, resultados, datos y estadísticas (1999: 352). Con respecto a la observación de nuestro análisis, y de acuerdo con la clasificación de Pérez Serrano, hemos realizado una 'observación externa o no participante directa', es decir, nos basamos en una investigación sobre el terreno, en contacto inmediato con la realidad, en nuestro caso, con la prensa deportiva digital española. Como indica la autora, "la principal ventaja de esta observación consiste en que el observador puede dedicar a ella toda su atención y realizar anotaciones a medida que se originan los fenómenos. Su mayor inconveniente es que no puede realzarse sin conocimiento de lo observado" (2008: 24-25).

Para garantizar la fiabilidad y validez del análisis, ciertas premisas deben ser respetadas. Varios autores indican cinco reglas esenciales (Bardin, 1986; Mayer y Quellet, 1991; Landry, 1998): a) La exhaustividad. Una vez que los contenidos a analizar están determinados, se deben considerar todos los elementos. La exhaustividad supone que las categorías establecidas permiten clasificar el conjunto del material recogido. b) La representatividad. Se puede garantizar cuando el material se presta para efectuar el análisis sobre la muestra. El muestreo es riguroso si constituye una parte representativa de todos los datos iniciales. c) La homogeneidad. Los documentos elegidos deben ser homogéneos, es decir escogidos en función de criterios precisos y no presentar mucha singularidad en relación a estos criterios. d) La pertinencia. Los documentos escogidos deben corresponder al objetivo del análisis. Una categoría es pertinente cuando ella hace posible el estudio del material obtenido ante las preguntas y el marco de análisis seleccionados. e) La univocación. Significa que una categoría tiene el mismo sentido para todos los investigadores.

Así, para este análisis cuantitativo, el primer paso ha consistido en la composición de unas tablas en la que hemos documentado los datos. Estas tablas se han dividido por diarios y meses; una global por diario y, además, una global del conjunto de todos los resultados totales de los diarios estudiados para obtener una visión integral. En estas tablas cuantitativas hemos contabilizado el número de contenidos informativos que se dedican a cada uno de los deportes.

En la primera tabla, estructurada por deportes en fútbol, baloncesto y motor, hemos creado subdivisiones, pues son los que más informaciones abarcan y tal subdivisión nos otorga una visión más específica y pormenorizada de la selección de la información de los medios analizados. En el caso del fútbol, esa subdivisión ha sido la siguiente: 
- $\quad$ Real Madrid

- Barcelona

- $\quad$ Atlético de Madrid

- $\quad$ Resto de Primera División

- Total de Primera División

- Resto Nacional

- $\quad$ Fútbol Internacional

- $\quad$ Total fútbol

En cada una de esas subdivisiones, hemos documentado numéricamente la cantidad de informaciones dedicadas a cada una de ellas. Sin embargo, cabe aclarar que en ciertos contenidos las informaciones aparecen entremezcladas; por ejemplo, aquellos que, a la vez, versen sobre Real Madrid y Barcelona. Para estos casos dudosos, hemos optado por el criterio de cuantificar tales contenidos en la casilla del equipo de la ciudad editora. Es decir, una crónica de un partido Real Madrid-Barcelona, para Marca y As se cuantificará en la casilla 'Real Madrid', y para Mundo Deportivo y Sport, en 'Barcelona'. En los casos dudosos entre Real Madrid y Atlético de Madrid, se considerarán para la casilla del primero, ya que, en términos generales, existe una preferencia (en número de contenidos) por el Real Madrid.

De la misma manera, informaciones relativas a los rivales de esos tres equipos que hemos diferenciado (Real Madrid, Barcelona y Atlético de Madrid) cuando se van a enfrentar o se han enfrentado a ellos. Por ejemplo, las declaraciones del entrenador del Eibar tras el partido ante el Atlético de Madrid, se acumularán en la casilla 'Atlético de Madrid' en lugar de 'Resto de Primera', pues entendemos que tales declaraciones se convierten en actualidad, de acuerdo con los criterios del medio, porque el rival es el Atlético de Madrid.

En el campo 'Resto Nacional' hemos cuantificado cualquier otro tipo de división inferior a Primera, así como las informaciones relativas a la Selección Nacional. Como excepción a este criterio, hemos optado por cuantificar las informaciones relativas a los equipos de categorías inferiores (y, si procede, en su categoría femenina) de Real Madrid, Barcelona y Atlético en las casillas de sus respectivos clubes, ya que, siguiendo con el criterio anterior, entendemos que son contenidos de actualidad por su pertenencia a esos clubes, pues no existe un seguimiento regular al mismo nivel con el resto de equipos de las respectivas categorías.

En cuanto a 'Fútbol internacional', además de cuantificar las informaciones relacionadas sobre fútbol más allá de nuestras fronteras (cualesquiera que sean sus categorías), hemos sumado las informaciones de las selecciones nacionales, 
aunque la información esté protagonizada por jugadores de la Liga española. Por ejemplo, un vídeo de la firma de un autógrafo de Cristiano Ronaldo a un niño durante la concentración de Portugal, se sumará a 'Fútbol internacional'.

Para la sección de Baloncesto, hemos dividido los contenidos del siguiente modo:

- $\quad \mathrm{ACB}$

- NBA

- Otros

- Total Baloncesto

En cuanto a los deportes de 'Motor', hemos establecido la siguiente subdivisión:

- $\quad$ Fórmula 1

- $\quad$ Motociclismo

- Otros

- Total Motor

Por último, hemos dividido otros dos de los deportes más populares: tenis y ciclismo; y el resto de informaciones relacionadas con el resto de deportes, las hemos categorizado en 'Otros'. Asimismo, hemos añadido otra casilla llamada 'Ocio y otros', en la que hemos contabilizado aquellos contenidos sin relación alguna con el deporte, pero que sí se publican en estos medios. De manera accesoria y para obtener un dato relevante, hemos añadido una casilla en la que al total de informaciones publicadas, restamos las informaciones de 'Total fútbol'. De este modo, conocemos el número y el porcentaje que se dedica a los deportes que no están relacionados con el mundo balompédico.

Nuestra investigación se ha basado en un seguimiento de los cuatro diarios deportivos nacionales más importantes por número de lectores (Marca, As, Sporty Mundo Deportivo) durante 61 días de forma no consecutiva, es decir, hemos decidido espaciarlo en el tiempo para, de ese modo, abarcar un periodo más dilatado y demostrar con este estudio que los resultados obtenidos no son fruto de la casualidad o de un evento específico que concentra la atención informativa durante un espacio de tiempo concreto. Así pues, es una muestra variada y azarosa, cuyo único objetivo consiste en basar la investigación en una muestra temporal lo suficientemente representativa. Los días de escrutinio de este análisis (todos correspondientes al año 2014) se dividen como sigue:

- $\quad$ Enero: del 14 al 24 (11 días)

- $\quad$ Febrero: del 9 al 18 (10 días) 
- $\quad$ Marzo: del 5 al 14 (10 días)

- $\quad$ Agosto: del 27 al 31 (5 días)

- $\quad$ Octubre: del 19 al 23 (5 días)

- $\quad$ Noviembre: del 15 al 24 (10 días)

- $\quad$ Diciembre: del 3 al 12 (10 días)

Como es evidente, es imposible realizar un estudio de esta magnitud sobre el número total de informaciones publicadas en un día en un diario digital, pues el número de contenidos resultaría inabarcable simplemente con las informaciones publicadas en la portada principal del diario, pero más inviable aún si tenemos en cuenta la cantidad de portadillas que subdividen el diario. Hemos optado por escoger una representación de las informaciones en función de la jerarquización de la portada principal, es decir, aquello a lo que el diario otorga mayor relevancia, por posición y tamaño. Así, se entiende que los contenidos de mayor enjundia (a juicio de la línea editorial de cada diario) son los que aparecen en posiciones de preferencia en la portada principal.

De esa portada principal, hemos decidido abarcar un número significativo de informaciones, con lo que hemos analizado las 30 primeras noticias de la portada de cada diario en cada uno de los días estudiados. De este modo, hemos examinado 1.830 contenidos de cada uno de los diarios, que, multiplicado por cada uno de ellos, nos arroja un total de 7.320 informaciones escrutadas, cantidad suficiente para establecer conclusiones sólidas sobre una muestra que consideramos altamente representativa.

En este punto, cabe indicar cómo hemos considerado la numeración de las posiciones de los contenidos. Los cuatro diarios se estructuran de una manera similar: en dos columnas (sin tener en cuenta la publicidad ni otros espacios promocionales destacados, normalmente en una tercera columna). Así, la primera posición será aquella situada en la zona superior izquierda; la segunda, la que aparece inmediatamente a la misma altura en la zona derecha; la tercera, la siguiente en la columna de la izquierda y así sucesivamente hasta contar 30 contenidos. Como decimos, hemos ignorado tanto los espacios publicitarios (tanto los que son anunciantes como los contenidos que difunden publicidad del propio diario, como promociones de productos, o secciones como Marca apuestas, etc.); ya que de no hacerlo así los resultados obtenidos perderían solidez. También hemos ignorado, por los mismos motivos y porque el estudio resultaría demasiado complejo en todos los sentidos, aquellos espacios destacados en los que se repiten informaciones, normalmente intercalados entre las secciones y sin ningún nuevo valor informativo. 


\section{Resultados}

Una vez desarrollada la metodología de análisis, los resultados obtenidos se han sistematizado en las tablas siguientes. La representación de los contenidos se ha dividido por periódicos y, para una visión global, hemos confeccionado una tabla conjunta en la que hemos agrupado todos los resultados. Esta estructuración nos permite presentar los datos de manera independiente para cada uno de los diarios y, además, mostrar la situación general de la prensa deportiva en España representada por los cuatro grandes diarios españoles a nivel nacional.

\subsection{Resultados globales}

En primer lugar, analizaremos los resultados obtenidos tras haber documentado de forma global los datos de los cuatro diarios. Ello nos aportará la perspectiva de cuál es la situación de la prensa deportiva española en Internet. Para obtener una perspectiva más visual de cuál es el reparto por deportes (siempre hablamos de las 30 primeras informaciones de las portadas analizadas), nos ayudaremos de un gráfico porcentual.

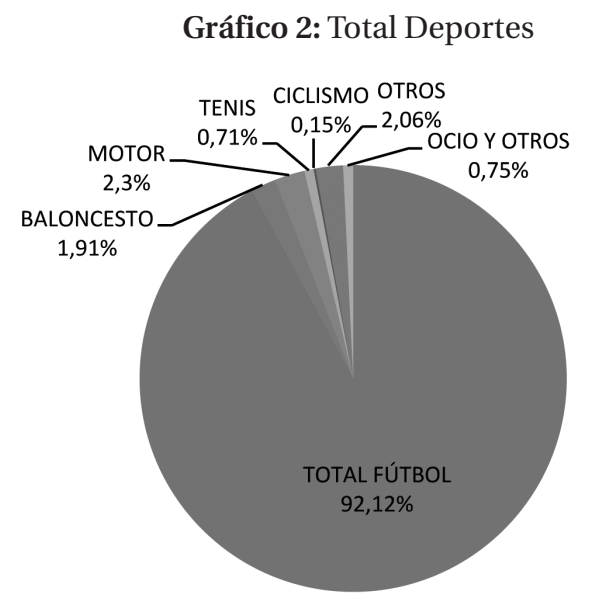

Fuente: Elaboración propia

Observamos que, de una manera muy destacada, el grueso de la información deportiva corresponde al fútbol, con un $92,12 \%$. Sobre el total, las informaciones relacionadas con el Barcelona (43,24\%) y el Real Madrid (22,01\%) ocupan un porcentaje del 65,25\%. La información total sobre fútbol de Primera División, suma el 78,88\% del total de contenidos; pero si ignoramos los relacionados con Barcelona y Real Madrid, esta cifra desciende hasta el 13,63\%, del cual, más de la mitad (el 7,28\%) se corresponden con informaciones sobre el Atlético de Madrid, lo que supondría solo un 6,35\% de la informa- 
ción total para el resto de los equipos de la máxima categoría. Si nos centramos en el resto de deportes, los vinculados con el mundo del motor ocupan el segundo puesto de la clasificación sobre aquellos a los que se dedica mayor atención con un 2,30\% del total de los contenidos. Dentro de este deporte, la fórmula 1 es la que tiene mejores resultados, con un 1,82\%. El motociclismo $(0,26 \%)$ y otros deportes de motor $(0,22 \%)$. La sección 'otros deportes' es la tercera en importancia en los datos globales con un $2,6 \%$, seguida del baloncesto $(1,91 \%)$, tenis $(0,71 \%)$ y ciclismo $(0,15 \%)$ completan los números de la actualidad deportiva en la prensa deportiva en nuestro país.

De la apreciación anterior se desprende que la información sobre fútbol monopoliza, con diferencia, la actualidad deportiva gracias a una preferencia muy acentuada hacia el Barcelona y el Real Madrid y, a una distancia considerable, hacia el Atlético de Madrid. De este modo, se observa que en la parcela futbolística la información está claramente seccionada, pues sólo el 6,89\% de la información sobre el total de fútbol se dedica a equipos que no son ni Barcelona, Real Madrid o Atlético, lo que muestra un notable desequilibrio informativo. Para comprender estos comentarios, observemos un nuevo gráfico sobre cómo se reparte la información exclusiva sobre fútbol.

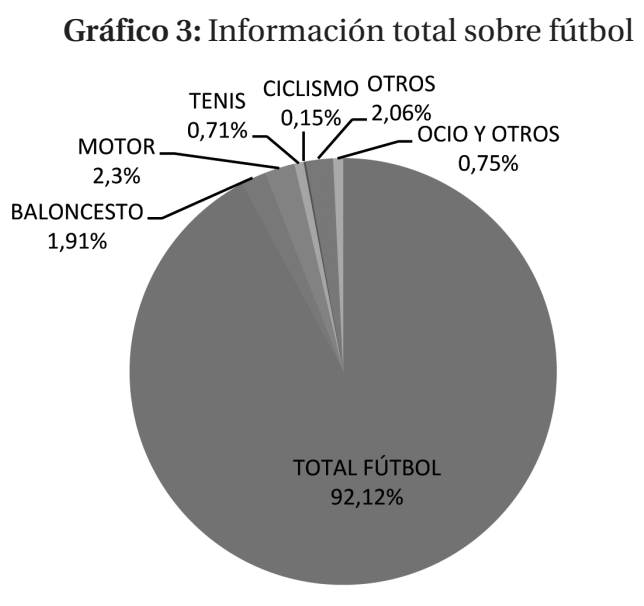

Fuente: Elaboración propia

Al fútbol internacional se le dedica un 9,7\% de la información total; y al resto del fútbol nacional (selección, resto de divisiones, fútbol femenino, etc.), un 3,54\%. Como consecuencia de esa preferencia hacia el mundo del balompié, el resto de los contenidos que no son fútbol representan sólo un 7,88\% de la actualidad deportiva, entre lo que se incluye un $0,75 \%$ que son contenidos de ocio (no relacionados con el deporte). 


\begin{tabular}{|c|c|c|c|c|c|c|c|c|}
\hline \multicolumn{9}{|c|}{ TABLA CUANTITATIVA / GLOBAL } \\
\hline & & & Marca & As & MD & Sport & TOTAL & $\%$ \\
\hline & \multirow{8}{*}{ FÚTBOL } & R. Madrid & 558 & 667 & 245 & 141 & 1611 & 22,01 \\
\hline & & F.C. Barcelona & 423 & 432 & 973 & 1337 & 3165 & 43,24 \\
\hline & & At. Madrid & 241 & 210 & 54 & 28 & 533 & 7,28 \\
\hline & & Resto $1^{\mathrm{a}}$ & 189 & 135 & 71 & 70 & 465 & 6,35 \\
\hline & & Total $1^{\mathrm{a}}$ & 1411 & 1444 & 1343 & 1576 & 5774 & 78,88 \\
\hline & & Resto nac. & 96 & 78 & 38 & 47 & 259 & 3,54 \\
\hline & & Fút. Inter. & 157 & 164 & 234 & 155 & 710 & 9,70 \\
\hline & & Total Fútbol & 1664 & 1686 & 1615 & 1778 & 6743 & 92,12 \\
\hline & \multirow{4}{*}{ BALONCESTO } & ACB & 14 & 31 & 29 & 10 & 84 & 1,15 \\
\hline & & NBA & 0 & 20 & 11 & 2 & 33 & 0,45 \\
\hline & & OTROS & 13 & 3 & 4 & 3 & 23 & 0,31 \\
\hline & & Total Baloncesto & 27 & 54 & 44 & 15 & 140 & 1,91 \\
\hline & \multirow{4}{*}{ MOTOR } & Fórmula 1 & 44 & 40 & 41 & 8 & 133 & 1,82 \\
\hline & & Motociclismo & 10 & 3 & 6 & 0 & 19 & 0,26 \\
\hline & & Otros & 5 & 6 & 3 & 2 & 16 & 0,22 \\
\hline & & TOTAL & 59 & 49 & 50 & 10 & 168 & 2,30 \\
\hline & TENIS & & 8 & 14 & 30 & 0 & 52 & 0,71 \\
\hline & CICLISMO & & 3 & 5 & 3 & 0 & 11 & 0,15 \\
\hline & OTROS & & 31 & 22 & 74 & 24 & 151 & 2,06 \\
\hline & OCIOY OTROS & & 38 & 0 & 14 & 3 & 55 & 0,75 \\
\hline TOTAL NO FÚTBOL & & & 166 & 144 & 215 & 52 & 577 & 7,88 \\
\hline TOTAL INFO & & & 1830 & 1830 & 1830 & 1830 & 7320 & 100,00 \\
\hline
\end{tabular}

Fuente: Elaboración propia

\subsection{Resultados por medio}

Una vez sumados los datos totales dedicados por los cuatro grandes diarios deportivos en sus ediciones de Internet a los distintos deportes, y especialmente al fútbol, creemos conveniente realizar un análisis pormenorizado de cómo se produce dicho reparto de los contenidos informativos en cada diario, pues pueden obtenerse resultados significativos y diferenciados sobre el equilibrio informativo seguido por cada medio. 


\subsubsection{Marca}

Siguiendo la estructura de análisis, el primer gráfico de la información publicada en el diario Marca distribuida por deportes produce los siguientes resultados.

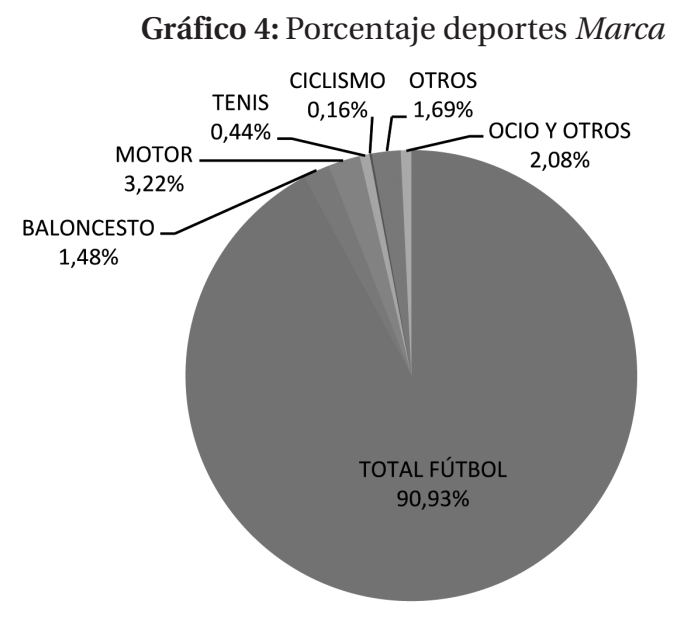

Fuente: Elaboración propia

Como primer dato altamente significativo comprobamos como el 90,93\% de la información se dedica al fútbol. El 77,1\% corresponde a contenidos de equipos de Primera División. En esa categoría, Real Madrid y Barcelona acaparan la atención informativa, pues se les dedica un 30,49\% y un 23,11\% del total de la actualidad deportiva, es decir, un 53,6\% de toda la información deportiva analizada trata sobre Real Madrid y Barcelona. Si le añadimos el espacio dedicado al Atlético de Madrid (13.17\%), el porcentaje asciende al 66,77\%. Todo el resto de equipos de Primera División se reparten un 10,33\% del total. El resto del fútbol nacional y la selección española obtienen el 5,25\% de los contenidos totales y el fútbol internacional, el 8,58\%. Los deportes de motor ocupan el segundo puesto de la clasificación sobre los deportes a los que Marca presta mayor atención, pero muy lejos del fútbol: un 3,22\% del total de los contenidos. Dentro de este deporte, la fórmula 1 es la que tiene mejores resultados con un $2,4 \%$. El motociclismo (0,55\%) y otros deportes de motor (0,27\%) completan el resto. Curiosamente, contenidos que no se relacionan directamente con la actividad deportiva (a los que hemos denominado como 'ocio y otros'), son los que copan la tercera categoría con más presencia en Marca, un 2,08\%. Suelen ser informaciones sobre cine, videojuegos, el mundo del espectáculo, etc. La sección 'otros deportes' (1,69\%), baloncesto $(1,48 \%)$, tenis $(0,44 \%)$ y ciclismo $(0,16 \%)$ completan los números de la actualidad deportiva de Marca. De este modo, la atención que se le presta a la información que no es fútbol supone el 9,07\% del total; una cifra que desciende al 6,99\% si no tenemos en cuenta el 2,08\% que corresponde a los contenidos de ocio. 
Por su parte, la atención informativa dedicada al deporte del balompié se reparte de la siguiente manera:

Gráfico 5: Porcentaje fútbol Marca

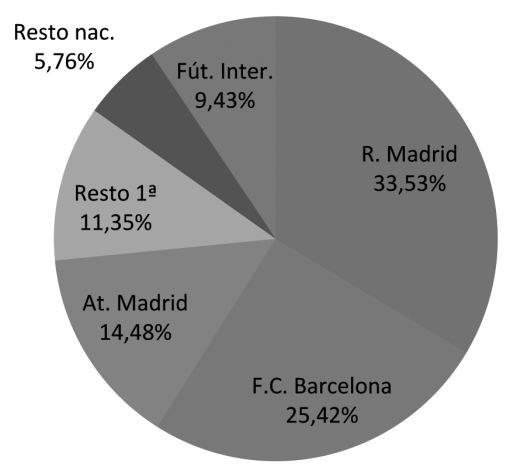

Fuente: Elaboración propia

Del total de la información futbolística, el 59\% corresponde a Real Madrid (33,53\%) y Barcelona (25,42\%). Sumado al 14,48\% que ocupa el Atlético de Madrid, resulta que estos tres equipos se reparten el 73,43\% de toda la atención sobre fútbol. Así, el $26,57 \%$ restante se reparte entre el resto de equipos de Primera División (11,35\%), el resto del fútbol nacional (5,76\%) y el fútbol internacional $(9,43 \%)$. 
Gráfico 6: Tabla resumen Marca

\begin{tabular}{|c|c|c|c|c|c|c|c|c|c|c|c|}
\hline & & & Ene. & Feb. & Mar. & Ago. & Oct. & Nov. & Dic. & Total & $\%$ \\
\hline & \multirow{8}{*}{ FÚTBOL } & R. Madrid & 108 & 76 & 82 & 37 & 49 & 77 & 129 & 558 & 30,49 \\
\hline & & F.C. Barcelona & 84 & 81 & 86 & 18 & 39 & 66 & 49 & 423 & 23,11 \\
\hline & & At. Madrid & 31 & 58 & 29 & 21 & 21 & 35 & 46 & 241 & 13,17 \\
\hline & & Resto $1^{\mathrm{a}}$ & 55 & 32 & 27 & 16 & 17 & 11 & 31 & 189 & 10,33 \\
\hline & & Total $1^{\mathrm{a}}$ & 278 & 247 & 224 & 92 & 126 & 189 & 255 & 1411 & 77,10 \\
\hline & & Resto nac. & 4 & 4 & 21 & 12 & 2 & 43 & 10 & 96 & 5,25 \\
\hline & & Fút. Inter. & 13 & 19 & 24 & 20 & 10 & 52 & 19 & 157 & 8,58 \\
\hline & & Total Fútbol & 295 & 270 & 269 & 124 & 138 & 284 & 284 & 1664 & 90,93 \\
\hline & \multirow{4}{*}{ BALONCESTO } & ACB & 5 & 4 & 2 & 0 & 0 & 2 & 1 & 14 & 0,77 \\
\hline & & NBA & 0 & 0 & 0 & 0 & 0 & 0 & 0 & 0 & 0,00 \\
\hline & & Otros & 0 & 0 & 0 & 13 & 0 & 0 & 0 & 13 & 0,71 \\
\hline & & \begin{tabular}{|l|} 
Total Balonc. \\
\end{tabular} & 5 & 4 & 2 & 13 & 0 & 2 & 1 & 27 & 1,48 \\
\hline & \multirow{4}{*}{ MOTOR } & Fórmula 1 & 10 & 4 & 11 & 0 & 0 & 8 & 11 & 44 & 2,40 \\
\hline & & Motociclismo & 3 & 0 & 3 & 0 & 4 & 0 & 0 & 10 & 0,55 \\
\hline & & Otros & 3 & 0 & 1 & 0 & 0 & 0 & 1 & 5 & 0,27 \\
\hline & & Total & 16 & 4 & 15 & 0 & 4 & 8 & 12 & 59 & 3,22 \\
\hline & TENIS & & 5 & 1 & 0 & 0 & 0 & 2 & 0 & 8 & 0,44 \\
\hline & CICLISMO & & 0 & 0 & 0 & 3 & 0 & 0 & 0 & 3 & 0,16 \\
\hline & OTROS & & 5 & 14 & 3 & 4 & 2 & 0 & 3 & 31 & 1,69 \\
\hline & OCIO Y OTROS & & 4 & 7 & 11 & 6 & 6 & 4 & 0 & 38 & 2,08 \\
\hline TOTAL NO FÚTBOL & & & 35 & 30 & 31 & 26 & 12 & 16 & 16 & 166 & 9,07 \\
\hline TOTAL INFO & & & 330 & 300 & 300 & 150 & 150 & 300 & 300 & 1830 & 100,00 \\
\hline
\end{tabular}

Fuente: Elaboración propia

\subsubsection{As}

Los porcentajes obtenidos de As se distribuyen de la siguiente manera 


\section{Gráfico 7: Porcentaje deportes As}

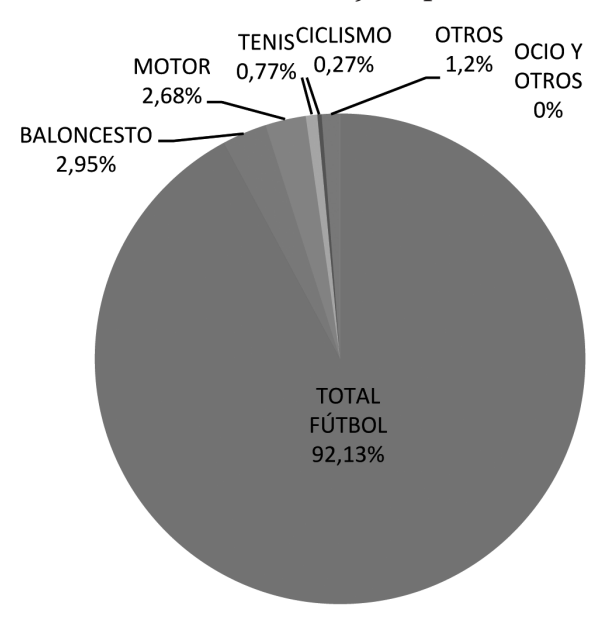

Fuente: Elaboración propia

Como también sucedía en la Marca, en la edición digital de As el 92,13\% de toda la información deportiva se dedica a informaciones sobre fútbol. En concreto, el 78,91\% se dedica a los equipos de Primera División. El 60,06\% de toda la información deportiva la acaparan contenidos relacionados con el Real Madrid (36,45\%) y el Barcelona (23,61\%). Si le sumamos el 11,48\% que se dedica al Atlético de Madrid, obtenemos una suma total del $71,54 \%$ de toda la actualidad deportiva. Sólo el 7,38\% se dedica al resto de equipos de la máxima categoría del fútbol español. El resto de la actualidad futbolística nacional (incluyendo a la Selección) ocupa un 4,26\% y el fútbol internacional, un 8,96\%. Los siguientes deportes a los que As presta más atención son el baloncesto y los deportes de motor, ambos con menos de un 3\% de la información total; un 2,95\% y un 2,68\% respectivamente. En la sección de motor, destaca el protagonismo de la fórmula 1, ya que de las todas informaciones sobre motor, esta disciplina en concreto representa el 81,63\% de los contenidos publicados (es decir, 40 de los 49 de motor). Algo parecido ocurre con el baloncesto, aunque en menor medida, donde las informaciones sobre la ACB, la competición nacional, suponen un $57,41 \%$ de los contenidos relacionados con ese deporte. Con menos del $1 \%$ de toda la información escrutada, nos encontramos con el tenis $(0,77 \%)$ y el ciclismo $(0,27 \%)$. La categoría 'otros', que incluye al resto de los deportes, suma un 1,2\%. De este modo, sólo el 7,87\% de los contenidos analizados son informaciones que no se dedican al fútbol.

Dentro de la categoría del fútbol, los contenidos se distribuyen del siguiente modo: 


\section{Gráfico 8: Total Fútbol $A s$}

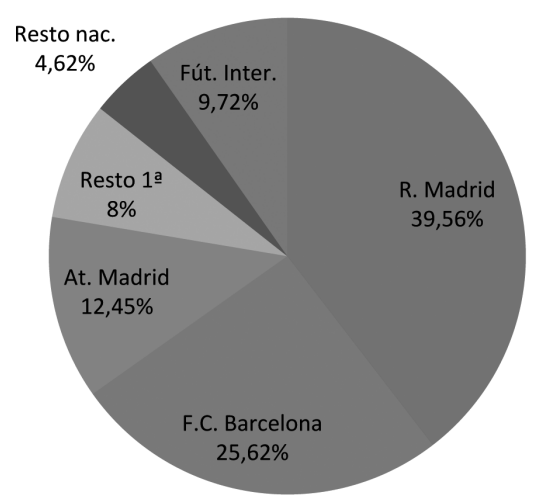

Fuente: Elaboración propia

Del total de informaciones sobre fútbol, el 77,63\% versan sobre la actualidad de Real Madrid, Barcelona y Atlético de Madrid. Los demás, se reparten el 22,37\% restante. Así, se observa que la supremacía sobre la actualidad futbolística la acaparan estos tres equipos, muy especialmente el Real Madrid (39\%) y el Barcelona (26\%). 
Gráfico 9: Tabla Deportes As

\begin{tabular}{|c|c|c|c|c|c|c|c|c|c|c|c|}
\hline & & & Ene. & Feb. & Mar. & Ago. & Oct. & Nov. & Dic. & Total & $\%$ \\
\hline & \multirow{8}{*}{ FÚTBOL } & R. Madrid & 125 & 103 & 101 & 53 & 56 & 104 & 125 & 667 & 36,45 \\
\hline & & F.C. Barcelona & 100 & 74 & 78 & 22 & 36 & 55 & 67 & 432 & 23,61 \\
\hline & & At. Madrid & 22 & 47 & 41 & 25 & 26 & 15 & 34 & 210 & 11,48 \\
\hline & & Resto $1^{\mathrm{a}}$ & 35 & 16 & 19 & 15 & 8 & 6 & 36 & 135 & 7,38 \\
\hline & & Total $1^{\mathrm{a}}$ & 282 & 240 & 239 & 115 & 126 & 180 & 262 & 1444 & 78,91 \\
\hline & & Resto nac. & 3 & 2 & 14 & 10 & 5 & 38 & 6 & 78 & 4,26 \\
\hline & & Fút. Inter. & 22 & 18 & 27 & 14 & 17 & 48 & 18 & 164 & 8,96 \\
\hline & & Total Fútbol & 307 & 260 & 280 & 139 & 148 & 266 & 286 & 1686 & 92,13 \\
\hline & \multirow{4}{*}{ BALONCESTO } & ACB & 4 & 17 & 6 & 0 & 2 & 2 & 0 & 31 & 1,69 \\
\hline & & NBA & 0 & 15 & 4 & 0 & 0 & 1 & 0 & 20 & 1,09 \\
\hline & & Otros & 1 & 0 & 0 & 0 & 0 & 0 & 2 & 3 & 0,16 \\
\hline & & Total Balonc. & 5 & 32 & 10 & 0 & 2 & 3 & 2 & 54 & 2,95 \\
\hline & \multirow{4}{*}{ MOTOR } & Fórmula 1 & 1 & 0 & 10 & 0 & 0 & 22 & 7 & 40 & 2,19 \\
\hline & & Motociclismo & 0 & 0 & 0 & 3 & 0 & 0 & 0 & 3 & 0,16 \\
\hline & & Otros & 6 & 0 & 0 & 0 & 0 & 0 & 0 & 6 & 0,33 \\
\hline & & Total & 7 & 0 & 10 & 3 & 0 & 22 & 7 & 49 & 2,68 \\
\hline & TENIS & & 5 & 0 & 0 & 0 & 0 & 9 & 0 & 14 & 0,77 \\
\hline & CICLISMO & & 0 & 0 & 0 & 5 & 0 & 0 & 0 & 5 & 0,27 \\
\hline & OTROS & & 6 & 8 & 0 & 3 & 0 & 0 & 5 & 22 & 1,20 \\
\hline & OCIO Y OTROS & & 0 & 0 & 0 & 0 & 0 & 0 & 0 & 0 & 0,00 \\
\hline TOTAL NO FÚTBOL & & & 23 & 40 & 20 & 11 & 2 & 34 & 14 & 144 & 7,87 \\
\hline TOTAL INFO & & & 330 & 300 & 300 & 150 & 150 & 300 & 300 & 1830 & 100,00 \\
\hline
\end{tabular}

Fuente: Elaboración propia

\subsubsection{Mundo Deportivo}

La atención informativa sobre el total de deportes por parte de Mundo Deportivo se estructura en los siguientes porcentajes: 


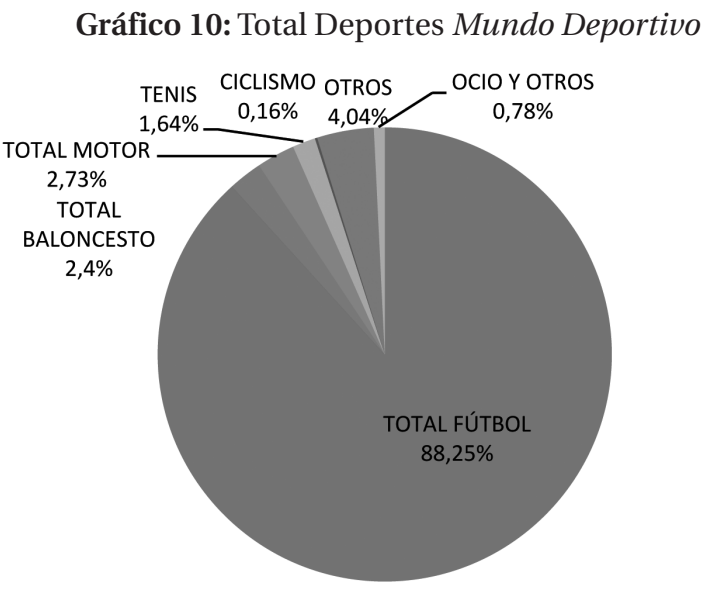

Fuente: Elaboración propia

Mundo Deportivo dedica el 88,25\% de sus principales informaciones al fútbol. El porcentaje que se destina a la máxima categoría ocupa el 73,39\% del total. De toda la información publicada en el diario, el 53,17\% son de contenidos relacionados con el Barcelona y el 13,39\%, con el Real Madrid. Así, entre ambos suman un total del 66,56\% de toda la información que publica el diario. La actualidad del fútbol internacional también ocupa un papel relevante en el diario deportivo decano, pues supone un 12,79\% de la información total. El Atlético de Madrid recibe un 2,95\% del total de la atención mediática de Mundo Deportivo y el todo resto de equipos de Primera División, el 3,88\%. Las demás categorías de este deporte, el fútbol femenino y la selección, es decir, el resto del fútbol nacional, tiene una presencia del 2,08\%. La sección 'otros deportes' es la segunda categoría más popular en el diario, con un 4,04\% y la que obtiene una cifra más alta en esta sección con respecto a los datos obtenidos en los otros tres diarios. Motor $(2,73 \%)$ y baloncesto $(2,4 \%)$ son los otros dos deportes de mayor relevancia en este caso. La sección de tenis sólo protagoniza el 1,64\% de las informaciones y el ciclismo, poco más del 0,16\% (en concreto, de los 1.830 contenidos, sólo 3 son sobre ciclismo). En total, la información que no versa sobre fútbol en Mundo Deportivo representa un 11,75\%.

Observemos, de manera gráfica, cómo se estructura la información futbolística en concreto: 


\section{Gráfico 11: Total Fútbol Mundo Deportivo}

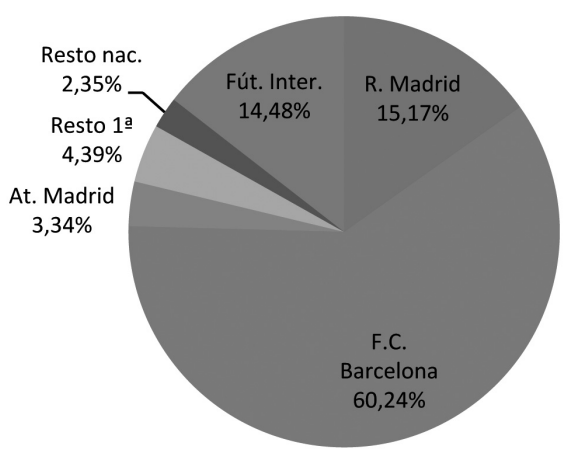

Fuente: Elaboración propia

El Barcelona ocupa claramente el centro de la actualidad deportiva, con un 60,24\% de los contenidos sobre fútbol. Si le sumamos el porcentaje del Real Madrid (15,17\%) obtenemos que tres cuartas partes de la información relacionada con el balompié se centra en estos dos equipos. Si a esos números le añadimos el porcentaje del fútbol internacional (14,48\%), obtenemos que el $89,89 \%$ de la información deportiva se basa en esas tres categorías y el 10,11\% restante se reparte entre el Atlético de Madrid (3,34\%), el resto de Primera División (4,39\%) y el resto del fútbol nacional (2,35\%). 
Gráfico 12: Cuadro resumen Mundo Deportivo

\begin{tabular}{|c|c|c|c|c|c|c|c|c|c|c|c|}
\hline & & & Ene. & Feb. & Mar. & Ago. & Oct. & Nov. & Dic. & Total & $\%$ \\
\hline & \multirow{8}{*}{ FÚTBOL } & R. Madrid & 39 & 38 & 55 & 24 & 7 & 35 & 47 & 245 & 13,39 \\
\hline & & F.C. Barcelona & 185 & 153 & 162 & 60 & 88 & 155 & 170 & 973 & 53,17 \\
\hline & & At. Madrid & 4 & 11 & 9 & 13 & 2 & 5 & 10 & 54 & 2,95 \\
\hline & & Resto $1^{\mathrm{a}}$ & 27 & 15 & 5 & 6 & 4 & 7 & 7 & 71 & 3,88 \\
\hline & & Total $1^{\mathrm{a}}$ & 255 & 217 & 231 & 103 & 101 & 202 & 234 & 1343 & 73,39 \\
\hline & & Resto nac. & 4 & 2 & 8 & 4 & 0 & 13 & 7 & 38 & 2,08 \\
\hline & & Fút. Inter. & 39 & 36 & 34 & 36 & 22 & 37 & 30 & 234 & 12,79 \\
\hline & & Total Fútbol & 298 & 255 & 273 & 143 & 123 & 252 & 271 & 1615 & 88,25 \\
\hline & \multirow{4}{*}{ BALONCESTO } & $\mathrm{ACB}$ & 3 & 8 & 5 & 1 & 4 & 5 & 3 & 29 & 1,58 \\
\hline & & NBA & 0 & 9 & 2 & 0 & 0 & 0 & 0 & 11 & 0,60 \\
\hline & & Otros & 1 & 0 & 0 & 2 & 0 & 0 & 1 & 4 & 0,22 \\
\hline & & Total Balonc. & 4 & 17 & 7 & 3 & 4 & 5 & 4 & 44 & 2,40 \\
\hline & \multirow{4}{*}{ MOTOR } & Fórmula 1 & 1 & 6 & 6 & 1 & 1 & 17 & 9 & 41 & 2,24 \\
\hline & & Motociclismo & 0 & 0 & 0 & 1 & 2 & 3 & 0 & 6 & 0,33 \\
\hline & & Otros & 2 & 0 & 0 & 0 & 1 & 0 & 0 & 3 & 0,16 \\
\hline & & Total & 3 & 6 & 6 & 2 & 4 & 20 & 9 & 50 & 2,73 \\
\hline & TENIS & & 15 & 0 & 2 & 0 & 6 & 7 & 0 & 30 & 1,64 \\
\hline & CICLISMO & & 0 & 0 & 1 & 0 & 2 & 0 & 0 & 3 & 0,16 \\
\hline & OTROS & & 7 & 18 & 11 & 2 & 9 & 16 & 11 & 74 & 4,04 \\
\hline & OCIO Y OTROS & & 3 & 4 & 0 & 0 & 2 & 0 & 5 & 14 & 0,77 \\
\hline TOTAL NO FÚTBOL & & & & & & & & & & 215 & 11,75 \\
\hline TOTAL INFO & & & 330 & 300 & 300 & 150 & 150 & 300 & 300 & 1830 & 100,00 \\
\hline
\end{tabular}

Fuente: Elaboración propia

\subsubsection{Sport}

En el diario Sport, la distribución porcentual de la información sobre Deportes se reparte de la siguiente manera: 
Gráfico 13: Total Deportes Sport

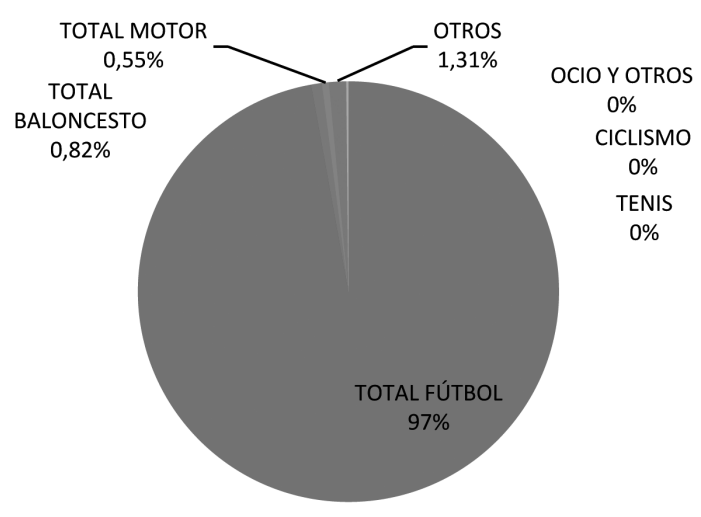

Fuente: Elaboración propia

Como se observa, los contenidos sobre fútbol monopolizan la atención informativa en Sport más que en ningún otro diario. El 97,16\% de los contenidos analizados de Sport se corresponden con este deporte. Cabe señalar que la web de Sport se estructura de un modo algo distinto con respecto al resto. Sport clasifica sus contenidos por secciones y la primera de ellas (que también es la más extensa) es la del Barcelona. Dicho esto, existe cierta flexibilidad, ya que cuando el diario lo considera oportuno, intercala informaciones en esa primera sección aunque hagan referencia a otras categorías o deportes. Debido a esa cantidad de fútbol, el resto de contenidos que no están vinculados a este deporte, obtienen un espacio del 2,84\% entre las 30 primeras informaciones de la portada. En concreto, las informaciones analizadas que no se dedican al fútbol suman 52 contenidos de los 1.830 .

Los deportes que no son fútbol, en su global, ocupan el siguiente espacio: el baloncesto supone el 0,82\% y los deportes de motor, el 0,55\% (ninguna de ellas sobre motociclismo). A lo largo de nuestra investigación no hemos encontrado un solo contendido relacionado con el tenis ni con el ciclismo. El resto de deportes tienen una presencia del 1,31\%. Si nos centramos en la sección de fútbol, hallamos que los contenidos que versan sobre la Primera División suponen un 86,12\% sobre el total de las informaciones publicadas. Este porcentaje viene dado debido al 73,06\% de la atención que se le presta al Barcelona. La segunda sección más popular en esta categoría es la de fútbol internacional, con un 8,47\% con respecto al total de informaciones; seguido por el Real Madrid, que supone el 7,7\% sobre el total de informaciones deportivas.

Si analizamos sólo la categoría de fútbol, las secciones se dividen del siguiente modo: 


\section{Gráfico 14: Total Fútbol Sport}

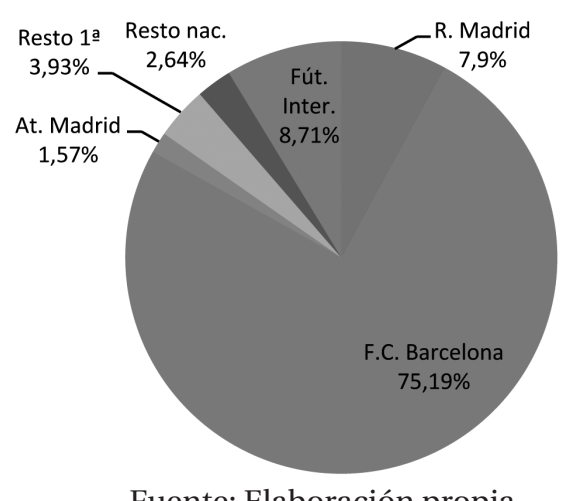

Fuente: Elaboración propia

Tres de cada cuatro noticias publicadas sobre fútbol, son acerca del Barcelona, es decir, el 75,19\%. Y es que, de las 1.830 noticias totales escrutadas, 1.337 están relacionadas con el club catalán. El fútbol internacional suma un 8,71\% y el Real Madrid, un 7,9\% con respecto a la información futbolística. 
Gráfico 15: Tabla resumen Sport

\begin{tabular}{|c|c|c|c|c|c|c|c|c|c|c|c|}
\hline & & & Ene. & Feb. & Mar. & Ago. & Oct. & Nov. & Dic. & Total & $\%$ \\
\hline & \multirow{8}{*}{ FÚTBOL } & R. Madrid & 24 & 18 & 30 & 16 & 12 & 19 & 22 & 141 & 7,70 \\
\hline & & F.C. Barcelona & 239 & 226 & 222 & 72 & 123 & 215 & 240 & 1337 & 73,06 \\
\hline & & At. Madrid & 3 & 10 & 4 & 4 & 1 & 4 & 2 & 28 & 1,53 \\
\hline & & Resto $1^{\mathrm{a}}$ & 20 & 14 & 10 & 17 & 3 & 4 & 2 & 70 & 3,83 \\
\hline & & Total $1^{\mathrm{a}}$ & 286 & 268 & 266 & 109 & 139 & 242 & 266 & 1576 & 86,12 \\
\hline & & Resto nac. & 6 & 6 & 7 & 8 & 4 & 13 & 3 & 47 & 2,57 \\
\hline & & Fút. Inter. & 30 & 18 & 24 & 29 & 3 & 31 & 20 & 155 & 8,47 \\
\hline & & Total Fútbol & 322 & 292 & 297 & 146 & 146 & 286 & 289 & 1778 & 97,16 \\
\hline & \multirow{4}{*}{ BALONCESTO } & ACB & 1 & 2 & 2 & 0 & 1 & 3 & 1 & 10 & 0,55 \\
\hline & & NBA & 0 & 0 & 0 & 0 & 0 & 0 & 2 & 2 & 0,11 \\
\hline & & Otros & 1 & 0 & 0 & 2 & 0 & 0 & 0 & 3 & 0,16 \\
\hline & & Total Balonc. & 2 & 2 & 2 & 2 & 1 & 3 & 3 & 15 & 0,82 \\
\hline & \multirow{4}{*}{ MOTOR } & Fórmula 1 & 0 & 1 & 0 & 0 & 0 & 5 & 2 & 8 & 0,44 \\
\hline & & Motociclismo & 0 & 0 & 0 & 0 & 0 & 0 & 0 & 0 & 0,00 \\
\hline & & Otros & 2 & 0 & 0 & 0 & 0 & 0 & 0 & 2 & 0,11 \\
\hline & & Total & 2 & 1 & 0 & 0 & 0 & 5 & 2 & 10 & 0,55 \\
\hline & TENIS & & 0 & 0 & 0 & 0 & 0 & 0 & 0 & 0 & 0,00 \\
\hline & CICLISMO & & 0 & 0 & 0 & 0 & 0 & 0 & 0 & 0 & 0,00 \\
\hline & OTROS & & 3 & 4 & 1 & 2 & 3 & 6 & 5 & 24 & 1,31 \\
\hline & OCIOY OTROS & & 1 & 1 & 0 & 0 & 0 & 0 & 1 & 3 & 0,16 \\
\hline TOTAL NO FÚTBOL & & & 8 & 8 & 3 & 4 & 4 & 14 & 11 & 52 & 2,84 \\
\hline TOTAL INFO & & & 330 & 300 & 300 & 150 & 150 & 300 & 300 & 1830 & 100,00 \\
\hline
\end{tabular}

Fuente: Elaboración propia

\section{Conclusiones}

Una vez realizada la tabulación de todos los datos obtenidos en el presente análisis cuantitativo de los principales diarios deportivos españoles en sus ediciones digitales, obtenemos como conclusión general el contundente dato de que el 91,12\% de las informaciones analizadas versan sobre fútbol. Esta conclusión confirma nuestra hipótesis inicial, evidente a ojos de cualquier lector de prensa deportiva en Internet, pero que hasta la actualidad no se había demostrado empíricamente mediante 
su cuantificación desde una perspectiva científica. Esto supone confirmar el objetivo general planteado en esta investigación, la constatación de la existencia de una sobreinformación sobre fútbol tanto en su cantidad como en la jerarquización de los contenidos relacionados con este deporte. Del mismo modo, se desprende que existe un silencio informativo o una infrainformación con respecto al resto de deportes, que tienen una presencia del 8,88\% de los 7.320 contenidos analizados. También podemos establecer, como una segunda conclusión que responde a la otra de los objetivos planteados que la atención informativa es protagonizada por los principales clubes de fútbol españoles: Barcelona y Real Madrid. Entre ambos, copan el $65,25 \%$ de toda la actualidad deportiva de nuestro análisis; el Barcelona, con un 43,24\% y el Real Madrid, un 22,01\%. Ésta es otra muestra de sobreinformación, así como de silencio informativo o infravaloración del resto de la actualidad deportiva.

La tercera conclusión obtenida, confirma también una de las hipótesis contempladas en los objetivos planteados, la parcialidad informativa con respecto a la información publicada sobre el equipo de la ciudad por parte de los diarios editados en la provincia; es decir, los medios catalanes prestan una atención especial al Barcelona y los madrileños, al Real Madrid. Esta parcialidad se acentúa considerablemente en el caso de los diarios catalanes, ya que si tenemos en cuenta sólo las informaciones publicadas con respecto a estos dos clubes, observamos que Marca y As mantienen cierto equilibrio: el Real Madrid supone el 58,8\%, mientras que el Barcelona, el 41,2\% restante. En el caso de Mundo Deportivo y Sport, en cambio, los porcentajes son más dispares: 14,3\% con respecto al Real Madrid; y un 85,7\% en cuanto al Barcelona. En concreto, es en el diario Sport donde esta diferencia se acentúa más, pues las informaciones sobre el Real Madrid representan un 9,5\%, mientras que las del Barcelona suponen un 90,5\%. Estos datos evidencian un trato informativo desequilibrado tanto entre estos dos equipos (parcialidad), como con respecto al resto de la actualidad deportiva (sobreinformación y silencio informativo).

Esta situación nos conduce a un aspecto sobre el que se ha discutido abundantemente en el ámbito periodístico, la relación entre información y audiencia, especialmente significativa en el ámbito de la prensa deportiva. Para Alcoba "existe una relación entre la demanda de los deportes solicitados por el público y la ofrecida por los medios, si bien se pierde, por esta motivación, la virtud de fomentar el deporte, valga la redundancia, como deporte simple y exclusivamente. El deporte tiene su importancia en los medios por el beneficio dejado al medio, pero no por otra cuestión" (1980: 278).

Para responder a esta hipótesis sobre la pérdida de calidad de la información deportiva por su derivación hacia el infoentretenimiento, la presente investigación se está completando en una siguiente fase con un estudio cuantitativo sobre los géneros informativos más empleados por la prensa deportiva en Internet, lo que nos ofrecerá qué tipo de contenidos y que tratamiento predomina en la información deportiva en Internet; se contempla también con un estudio cualitativo sobre las principales disfunciones de la prensa deportiva, entendida según la última edición del DRAE como "desarreglo en el funcionamiento de algo o en la función que le corresponde”. Para ello nos basaremos en la doctrina científica sobre la materia, los principales Códigos Deontológicos nacionales e internacionales y los Libros de Estilo de los medios de comunicación más representativos. 


\section{Referencias bibliográficas}

AEDE (2014): Libro Blanco de la Prensa 2014. Madrid: AEDE.

Alcoba, A. (1980): El periodismo deportivo en la sociedad moderna. Madrid: Hijos de E. Minuesa.

Alcoba, A. (1988): Especialización: Futuro del Periodismo. Madrid: Caja de Ahorros y Monte de Piedad de Madrid.

Alcoba, A. (1999): La prensa deportiva. Madrid: Instituto Universitario Olímpico de las Ciencias del Deporte.

Alcoba, A. (2005): Periodismo deportivo. Madrid: Síntesis.

Altabella, A (1997): "Historia de la prensa deportiva madrileña”, en Zabalza, R. (coord.): Orígenes del deporte madrileño. Madrid: Consejería de Educación, Dirección General de Deportes, pp. 169-226.

Artells, J. M. (1996): “Esports i mitjans de comunicació”, en Jones, D. E. (ed.): Esports i mitjans de comunicació a Catalunya. Barcelona: Generalitat de Catalunya. pp. 150-161.

Bardin, L. (1986): El análisis de contenido. Madrid: Akal.

Barrero, J. (2008): Periodistas deportivos: contra la violencia en el fútbol, al pie de la letra. Madrid: Fragua.

Barrero, J. (2009): Protagonistas contra la violencia en el deporte. Madrid: Fragua.

Berrocal, S. y Rodríguez-Maribona, C. (1998): Análisis Básico de la Prensa Diaria. Madrid: Universitas.

Berrocal, S. y García, E. (2001): “Comunicación e Información en Internet”, en Galdón, G. (coord.): Introducción a la comunicación y a la información. Barcelona: Ariel, pp. 229-251.

Campbell, D. T. y Stanley, J. C. (1996): Experimental and quasi-experimental designs of research. Chicago: Rand McNally \& Company.

Castañón, J. (1993): El lenguaje periodístico del fútbol. Valladolid: Universidad de Valladolid.

Castañón, J. (2004): “Magia y fiesta del lenguaje deportivo”, Comunicación y Estudios Universitarios, n. 12, pp. 51-58.

Cea, M. A. (1999): Metodología cuantitativa. Estrategias y técnicas de investigación social. Madrid: Síntesis.

Díaz Noci, J. y Meso, K. (1999): Periodismo en Internet. Bilbao: Universidad del País Vasco.

Duverger, M. (1974): Métodos de las ciencias sociales. Barcelona: Ariel.

Jones, D.E. (Ed.) (1996): Esports i mitjans de comunicació a Catalunya. Barcelona: Generalitat de Catalunya.

Esteve, F. y Fernández del Moral, J. (1999): Áreas de especialización periodística. Madrid: Fragua.

Ferman, G. S. y Levin J. (1979): Investigación en las ciencias sociales. México: Limusa.

García Candau, J. (1999): “Deportes”, en Amorós, A. y Díez Borque, J. M. (coords.): Historia de los espectáculos en España. Madrid: Castalia, pp. 529-539. 
Grinell, R. M. (2004): Social Work research \& evaluation: Quantitative and qualitative approaches. Oxford: Oxford University Press.

Landry, R. (1998): “L’analyse de contenu”, en Gauthier, B. (ed.): Recherche sociale. De la problemátique à la collecte des données. Quebec: Presses de l'Université du Québec, pp. 319-356.

Lázaro Carreter. F. (1994): “El español en el lenguaje deportivo”, en Reigosa, C. A. et al (coords.): El idioma español en el deporte. Madrid: Fundación EFE, pp. 19-36.

Hernández Alonso, N. (2003): El lenguaje de las crónicas deportivas. Madrid: Cátedra.

Herrero, C. (2006): “Periodismo deportivo y espacio público: estrategias de diseño, lenguaje y tratamiento informativo”, en Fernández, J. J., Sanz, C. y Rubio, A. L. (coords.): Prensa y periodismo especializado 3, vol. I. Guadalajara: Asociación de la Prensa de Guadalajara, pp. 137-151.

Hernández, R., Hernández, C. y Baptista, P. (2008): Metodología de la investigación. México: McGraw-Hill.

Gil, E. y Romero, H. (2012): “Consideraciones sobre la radio deportiva actual en España. Nuevos hábitos y horizontes”, en Marín Montín, J. (coord.): Deporte, comunicación y cultura. Zamora: Comunicación Social.

Mayer, R. y Quellet, F. (1991): Méthodologie de recherche pour les interventants sociaux. Montreal-Paris-Casablanca: Boucherville-Gäetan Morin Editeur.

Núñez Ladevéze, L. (1991): Manual para periodismo. Barcelona: Ariel.

Padua, J. (2000): Técnicas de investigación aplicadas a las ciencias sociales. México: Fondo de Cultura Económica.

Pérez Serrano, G. (2008): Investigación cualitativa. Retos e interrogantes. II Técnicas y análisis de datos. Madrid: La Muralla.

Relaño, A. (2004): “La prensa deportiva”, en Corell, M. et al. (coords.): Doce calas en la Historia de la prensa española especializada. Guadalajara: Editores del Henares C. B, pp. 331-367.

Rojas Soriano, R. (2001): Guía para realizar investigaciones sociales. México: Plaza y Valdés.

Rojas Torrijos, J. L. (2011): Periodismo deportivo de calidad. Madrid: Fragua.

Sierra, R. (1991): Técnicas de investigación social. Madrid: Paraninfo.

Sobrados, M. (2009): “La crónica de fútbol: evolución, estructura y lenguaje”, en Fernández, J. J., Sanz, C. y Rubio, A. L. (coords.): Prensa y periodismo especializado 4, Volumen II. Guadalajara: Asociación de la Prensa de Guadalajara, pp. 79-90.

Toro, C. (2008): La Historia de Marca (1938-2008). Madrid: La Esfera de los Libros.

Warren, C. N. (1975): Géneros periodísticos informativos. Barcelona: A.T.E. 